\title{
Malignant Pleural Effusion from Lung Adenocarcinoma Treated by Gefitinib
}

\author{
Akihito Kubo ${ }^{1,2}$, Yasuhiro $\mathrm{Koh}^{3}$, Tomoya Kawaguchi ${ }^{1}$, Shun-Ichi Isa ${ }^{1}$, Isamu Okamoto ${ }^{4}$, \\ Junya Fukuoka ${ }^{5}$, Yoko Kusunoki ${ }^{1}$, Masanori Kitaichi ${ }^{1}$, \\ Minoru Takada ${ }^{1}$ and Kazuhiko Nakagawa ${ }^{4}$
}

\begin{abstract}
Small molecule inhibitors targeting epidermal growth factor receptor (EGFR) are known to be active against non-small cell lung cancer (NSCLC) although the pharmacodynamics of these agents on malignant pleural effusion (MPE) remains unclear. Here we describe a case of lung adenocarcinoma with massive MPE treated successfully by gefitinib and chest drainage. Using sequential MPE samples before and during gefitinib therapy, the morphological changes and apoptosis of cancer cells were analyzed. Apoptosis of cancer cells was detected as early as 4 hours on, but not before, gefitinib therapy, suggesting that the pharmacodynamic assessment of such molecular targeting agents might be feasible for MPE.
\end{abstract}

Key words: lung cancer, adenocarcinoma, malignant pleural effusion, pharmacodynamics, gefitinib

(Intern Med 50: 745-748, 2011)

(DOI: 10.2169/internalmedicine.50.4652)

\section{Introduction}

Malignant pleural effusion (MPE) occurs in $10 \%$ to $50 \%$ of patients during the course of non-small cell lung cancer (NSCLC), most frequently adenocarcinoma $(1,2)$; the prognosis of symptomatic MPE is often dismal with a median survival of less than four months (3). Epidermal growth factor receptor tyrosine kinase inhibitors (EGFR TKIs), gefitinib or erlotinib, are active against NSCLC, especially those harboring EGFR mutations $(4,5)$ but the efficacy of these agents on MPE remains unclear. While the pharmacodynamics of EGFR TKIs have been reported in several cancers (6-10), the very early response of cancer cells to these drugs in vivo has not been described. Here we show a case of lung adenocarcinoma with massive malignant pleural effusion, successfully treated by gefitinib and by chest drainage, and describe the tumor cell response to gefitinib using serially sampled malignant effusions.

\section{Case Report}

A 67-year-old man with stage IV lung adenocarcinoma was referred to our hospital for further evaluation and treatment for his lung cancer. He had already received 2 lines of chemotherapy regimens after postoperative recurrence; carboplatin plus paclitaxel for 3 months and gefitinib as second line chemotherapy for 20 months. Gefitinib had been ended because of progression of bone metastases and right pleural dissemination 3 months prior to the referral to our hospital. Chest X-ray showed massive pleural effusion in the right thorax (Fig. 1A). As he seemed to have no indication for cytotoxic chemotherapy because of poor general condition with performance status 3 and the disease seemed to have progressed rapidly after the cessation of gefitinib, he received gefitinib again concurrently with chest drainage. He responded well to gefitinib and the chest drain was removed on day 12 on gefitinib treatment with no pleurodesis. His

\footnotetext{
${ }^{1}$ National Hospital Organization Kinki-Chuo Chest Medical Center, Japan, ${ }^{2}$ Division of Respiratory Medicine and Allergology, Department of Internal Medicine, Aichi Medical University School of Medicine, Japan, ${ }^{3}$ Drug Discovery and Development Division, Shizuoka Cancer Center Research Institute, Japan, ${ }^{4}$ Department of Medical Oncology, Kinki University School of Medicine, Japan and ${ }^{5}$ Department of Pathology, Toyama University School of Medicine, Japan

Received for publication October 8, 2010; Accepted for publication December 6, 2010

Correspondence to Dr. Akihito Kubo, kuboa@aichi-med-u.ac.jp
} 


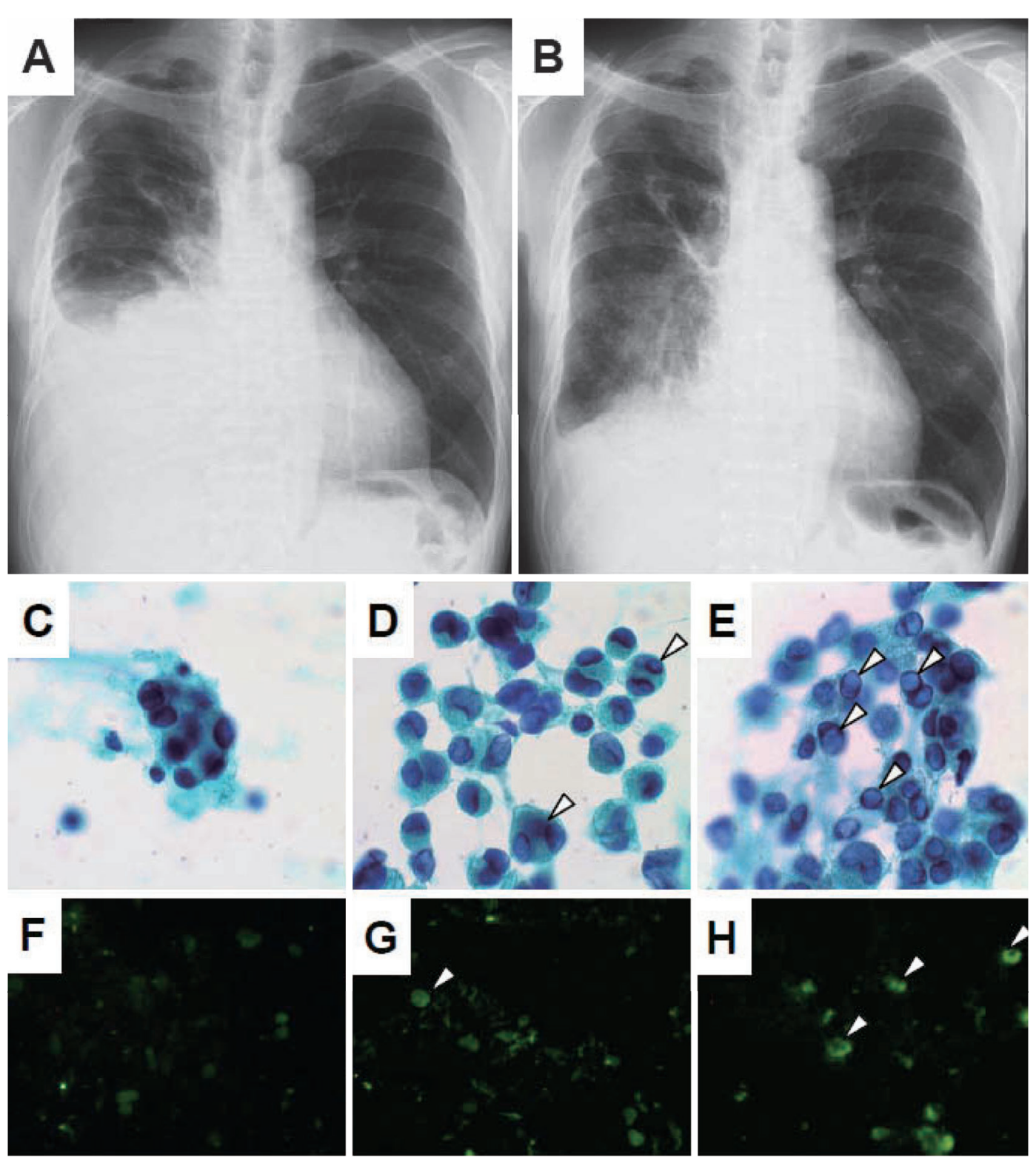

Figure 1. A and B: Chest X-rays on admission before re-treatment with gefitinib and chest drainage (A), and 2 months on therapy (B). C-H: Papanicolaou staining (C-E) and TUNEL staining (F-H) of malignant pleural effusion from case no. 1 before treatment and during re-treatment with gefitinib monotherapy. Pleural effusion $(50 \mathrm{~mL})$ was serially collected through an indwelling chest drainage tube before (C and F) and $4 \mathrm{~h}(\mathrm{D}$ and $\mathrm{G})$ and $24 \mathrm{~h}$ (E and H) after initiation of gefitinib (magnification: C-E, $\times 400$; F-H, $\times 200$ ).

general condition recovered to performance status 1 and he discharged from the hospital on day 29 on gefitinib treatment. He successfully continued gefitinib re-treatment for further 2 months (Fig. 1B) until disease progression.

We examined his pleural effusion before restarting gefitinib for gene mutations in EGFR and K-ras by peptide nucleic acid (PNA)-locked nucleic acid (LNA) PCR clamp assay using DNA extracted from ethanol-fixed cytology samples (11). The tumor harbored 2 EGFR mutations, an L858R gefitinib-sensitive mutation and a trace level of T790M gefitinib-resistant mutation. During gefitinib treatment, pleural effusion was sampled through the chest tube and examined for morphological changes and for apoptosis. We observed a fraction of cells as early as 4 hours on treatment, not before gefitinib, with chromatin condensed under the nuclear membrane, appearing as dense granular hemilunar caps, indicating cells undergoing apoptosis (Fig. 1C-E, arrowheads). These apoptotic cells were positively stained with the terminal transferase dUTP nick end labeling (TUNEL) technique (Fig. 1F-H, arrowheads). The mean fractions of apoptotic cells on 3 random high-power-fields before, $4 \mathrm{~h}$ on, and $24 \mathrm{~h}$ on gefitinib treatment were $0 \%$, $8.6 \%$, and $16.4 \%$, respectively on Papanicolaou staining, and $0.7 \%, 5.8 \%$, and $13.3 \%$, respectively on TUNEL staining. These assays were done upon the written informed consent from this patient and the approval of the institutional review board.

To examine the safety and efficacy of gefitinib on MPE 
Table 1. Characteristics and Treatment Outcome of Patients with Malignant Pleural Effusion from Non-small Cell Lung Cancer

\begin{tabular}{|c|c|c|c|c|c|c|c|c|c|c|c|}
\hline \multirow[t]{2}{*}{$\#$} & \multirow{2}{*}{$\begin{array}{c}\text { Gender/ } \\
\text { Age }\end{array}$} & \multirow[t]{2}{*}{ Histology } & \multirow{2}{*}{$\begin{array}{c}\text { Smoking } \\
\text { (pack-years) }\end{array}$} & \multirow{2}{*}{ PS } & \multirow{2}{*}{$\begin{array}{l}\text { \# prior } \\
\text { chemo. }\end{array}$} & \multicolumn{2}{|c|}{ Response to gefitinib* } & \multirow{2}{*}{$\begin{array}{l}\text { TTF } \\
\text { (mos) }\end{array}$} & \multirow{2}{*}{$\begin{array}{c}\text { Survival } \uparrow \\
\text { (mos) }\end{array}$} & \multicolumn{2}{|c|}{ Mutation status } \\
\hline & & & & & & Tumor & MPE & & & EGFR & K-ras \\
\hline 1 & $\mathrm{M} / 67$ & Ad & ES (25) & 3 & 2 & NTL & PR & 3.0 & $4.0 \mathrm{~L}$ & $\mathrm{~L} 858 \mathrm{R}+\mathrm{T} 790 \mathrm{M}$ & wildtype \\
\hline 2 & $\mathrm{~F} / 58$ & Ad & CS (46) & 1 & 1 & NTL & PR & 19.0 & 20.0 & wild type & ND \\
\hline 3 & $\mathrm{~F} / 52$ & Ad & NS & 1 & 2 & PR & NR & 18.1 & 18.2 & ND & ND \\
\hline 4 & $\mathrm{~F} / 56$ & Ad & NS & 1 & 1 & SD & PR & 9.6 & 13.9 & wild type & ND \\
\hline 5 & $\mathrm{~F} / 81$ & Ad & NS & 4 & 0 & SD & NR & 5.5 & 6.5 & L858R & ND \\
\hline 6 & $\mathrm{M} / 57$ & Ad & $\mathrm{ES}(72)$ & 3 & 2 & PD & NR & 1.0 & 1.1 & wild type & wildtype \\
\hline 7 & $\mathrm{M} / 61$ & Ad & ES (46) & 3 & 1 & PD & NR & 0.2 & 0.5 & wild type & wildtype \\
\hline
\end{tabular}

PS, performance status (ECOG); MPE, malignant pleural effusion; TTF, time to treatment failure; F, female; M, male; Ad, adenocarcinoma; S, current smoker; NS, never smoker; ES, ex smoker; NTL, no target lesions; SD, stable disease; PR, partial response; PD, progressive disease; NR, no response; ND, not determined. * Anti-tumor response was evaluated by the Response Evaluation Criteria in Solid Tumors. Response of MPE was judged according to the response evaluation criteria of malignant pleural effusion with local treatment by the Japan Lung Cancer Society (12), defined as: 1) complete response (disappearance of pleural effusion for 4 weeks or longer); 2) partial response (marked reduction of pleural effusion for 4 weeks or longer); 3) no response (not met 1) or 2)). $\$$ calculated as the time from gefitinib initiation to discontinuation of therapy. If calculated as the time from gefitinib initiation to death from any cause.

from NSCLC, we retrospectively reviewed 180 consecutive NSCLC patients treated by gefitinib in our hospital from the time of the approval of gefitinib use in Japan in 2002 to the present patient, following the approval of the survey by the institutional review board. Among these 180 patients, 7 adenocarcinoma patients, including the present patient (no. 1), had a massive MPE, defined as a pleural effusion greater than one-third of hemithorax (Table 1). In 5 patients, gefitinib was continued for $\geq 3$ months, and pleural effusion was well controlled for $\geq 5$ months in 4 patients (12). No adverse grade 3 or 4 effects defined by NCI-CTC version 2 and no interstitial lung disease were observed.

\section{Discussion}

Pharmacodynamic studies may offer valuable information on the effectiveness of targeted agents, and identify potential markers of clinical benefit. The pharmacodynamics of gefitinib or erlotinib have been reported on normal skin and several cancers using paired or sequential tumor biopsy samples before and during treatment, and it has been shown to be feasible to assess biological responses in the serial biopsy samples (6-8). However, there are several limitations to examine the biological responses in the tumor: 1) conducting sequential biopsy is difficult, 2) assessing very early responses in tumor tissues, e.g., several hours on therapy, is particularly challenging, 3) safety concerns remain in obtaining tumor tissues (8). The favorable clinical course of the present case suggests the feasibility of sequential MPE sampling during gefitinib treatment with concurrent chest drainage. The treatment outcome of the other 6 cases (Table 1) also supports the feasibility of gefitinib for patients with massive MPE. MPE may be a suitable condition for pharmacodynamic assessment of molecular targeting agents due to the ease of repetitive sampling of tumor cells from pleural fluids using an indwelling chest tube. Tube thoracostomy may be difficult during cytotoxic chemotherapy which has hematologic toxicities, leading to an increased risk of infections due to leucopenia/neutropenia and a risk of bleeding due to thrombocytopenia (13). This is the first report, within the scope of our literature search, which describes the very early induction of apoptosis in vivo by gefitinib treatment. While drug concentrations in the blood or pleural effusion were not examined in this study, we speculate that small molecule gefitinib might distribute to pleural effusion and reach the therapeutic concentration level, considering the rapid distribution of gefitinib with 3-7 hours of the time to maximum blood concentration (14). The tumor of this patient responded relatively well to retreatment gefitinib despite the presence of gefitinib-resistant T790M mutation. While L858R sensitive mutation was readily detectable by PNA-LNA PCR clamp assay in this patient's pleural effusion sample, T790M was at first undeterminable. By the reexamination of the assay $\mathrm{T} 790 \mathrm{M}$ was detected at the very lower limit of the detection level. Therefore the vast majority of mutant EGFR molecules may still have been L858R in this case and it may have taken 3 months, the time to treatment failure in this patient, for the minor clone T790M to expand and clinically acquire gefitinib resistance. From these observations we hypothesized that intensive pharmacokinetic and pharmacodynamic assessments of molecular targeting agents are feasible using serial sampling of malignant pleural fluids, on the condition that the agents have activities on these serious diseases, and have less hematologic toxicities. To test this hypothesis we have set up pharmacokinetic, pharmacodynamic and phase II studies of gefitinib and erlotinib in patients with malignant pleural effusion from non-small cell lung cancer.

\section{The authors state that they have no Conflict of Interest (COI).}

\section{Acknowledgement}

We thank Tomoaki Teramoto, Yoshio Fujita and Naoyuki Tajima for preparing pathology samples, and Juri Mori for data management. The work was supported in part by a Grant-in-Aid for Cancer Research (16-15) from the Ministry of Health, Labour and Welfare. 


\section{References}

1. Fenton KN, Richardson JD. Diagnosis and management of malignant pleural effusions. Am J Surg 170: 69-74, 1995.

2. Sekine I, Sumi M, Saijo N. Local control of regional and metastatic lesions and indication for systemic chemotherapy in patients with non-small cell lung cancer. Oncologist 113 Suppl: 21-27, 2008.

3. Burrows CM, Mathews WC, Colt HG. Predicting survival in patients with recurrent symptomatic malignant pleural effusions: an assessment of the prognostic values of physiologic, morphologic, and quality of life measures of extent of disease. Chest 117: 73$78,2000$.

4. Heist RS, Christiani D. EGFR-targeted therapies in lung cancer: predictors of response and toxicity. Pharmacogenomics 10: 59-68, 2009.

5. Sharma SV, Bell DW, Settleman J, Haber DA. Epidermal growth factor receptor mutations in lung cancer. Nat Rev Cancer 7: 169$181,2007$.

6. Albanell J, Rojo F, Averbuch S, et al. Pharmacodynamic studies of the epidermal growth factor receptor inhibitor ZD1839 in skin from cancer patients: histopathologic and molecular consequences of receptor inhibition. J Clin Oncol 20: 110-124, 2002.

7. Baselga J, Albanell J, Ruiz A, et al. Phase II and tumor pharmacodynamic study of gefitinib in patients with advanced breast cancer. J Clin Oncol 23: 5323-5333, 2005.

8. Felip E, Rojo F, Reck M, et al. A phase II pharmacodynamic study of erlotinib in patients with advanced non-small cell lung cancer previously treated with platinum-based chemotherapy. Clin Cancer Res 14: 3867-3874, 2008.

9. Rojo F, Tabernero J, Albanell J, et al. Pharmacodynamic studies of gefitinib in tumor biopsy specimens from patients with advanced gastric carcinoma. J Clin Oncol 24: 4309-4316, 2006.

10. Rothenberg ML, LaFleur B, Levy DE, et al. Randomized phase II trial of the clinical and biological effects of two dose levels of gefitinib in patients with recurrent colorectal adenocarcinoma. J Clin Oncol 23: 9265-9274, 2005.

11. Nagai $Y$, Miyazawa H, Huqun, et al. Genetic heterogeneity of the epidermal growth factor receptor in non-small cell lung cancer cell lines revealed by a rapid and sensitive detection system, the peptide nucleic acid-locked nucleic acid PCR clamp. Cancer Res 65: 7276-7282, 2005.

12. The Japan Lung Cancer Society. General Rule for Clinical and Pathological Record of Lung Cancer. The 5th edition. Kanehara \& Co., Ltd., Tokyo, 1999 (in Japanese).

13. Liou SY, Stephens JM, Carpiuc KT, Feng W, Botteman MF, Hay JW. Economic burden of haematological adverse effects in cancer patients: a systematic review. Clin Drug Investig 27: 381-396, 2007.

14. Nakagawa K, Tamura $T$, Negoro $S$, et al. Phase I pharmacokinetic trial of the selective oral epidermal growth factor receptor tyrosine kinase inhibitor gefitinib ('Iressa', ZD1839) in Japanese patients with solid malignant tumors. Ann Oncol 14: 922-930, 2003.

(C) 2011 The Japanese Society of Internal Medicine http://www.naika.or.jp/imindex.html 